Article

\title{
An Exact Efficiency Formula for Holographic Heat Engines
}

\author{
Clifford V. Johnson
}

Department of Physics and Astronomy, University of Southern California, Los Angeles, CA 90089-0484, USA; johnson1@usc.edu; Tel.: +1-213-821-1772; Fax: +1-213-740-6653

Academic Editor: David Kubiznak

Received: 10 February 2016; Accepted: 28 March 2016; Published: 31 March 2016

\begin{abstract}
Further consideration is given to the efficiency of a class of black hole heat engines that perform mechanical work via the $p d V$ terms present in the First Law of extended gravitational thermodynamics. It is noted that, when the engine cycle is a rectangle with sides parallel to the $(p, V)$ axes, the efficiency can be written simply in terms of the mass of the black hole evaluated at the corners. Since an arbitrary cycle can be approximated to any desired accuracy by a tiling of rectangles, a general geometrical algorithm for computing the efficiency of such a cycle follows. A simple generalization of the algorithm renders it applicable to broader classes of heat engine, even beyond the black hole context.
\end{abstract}

Keywords: black holes; thermodynamics; heat engines

\section{Introduction}

This paper concerns the efficiency of holographic heat engines, which were defined in [1]. They are a natural concept in extended gravitational thermodynamics, which, in making dynamical the cosmological constant $(\Lambda)$ in a theory of gravity, supplies (here we are using geometrical units where $G, c, h, k_{\mathrm{B}}$ have been set to unity) a pressure variable $p=-\Lambda / 8 \pi$ and its conjugate volume $V$ (see [2-13]).

One may extract mechanical work via the $p d V$ term in the First Law of Thermodynamics, and so it is possible to define a cycle in state space during which there is a net input heat $Q_{H}$ flow, a net output heat flow $Q_{C}$, and a net output work $W$ such that $Q_{H}=W+Q_{C}$. See Figure 1a. The efficiency is then $\eta=W / Q_{H}=1-Q_{C} / Q_{H}$. Its value is determined by the equation of state of the system and the choice of cycle in state space. The gravitational solution (a black hole, in the cases studied here) supplies the equation of state: The temperature $T$, entropy $S$, and other quantities can be computed [14-17], and there are relations between them. There's also a relation between the thermodynamic volume $V$ and the horizon radius of the black hole [2]. The precise form of all these relations depends upon the type of black hole, and of course the parent theory of gravity under discussion. For example, $[18,19]$ study the efficiency in the situation when the parent gravity theory has Gauss-Bonnet and Born-Infeld sectors.

In this extended thermodynamics context, we work with a negative cosmological constant (defining a positive pressure), for which such physics has an holographic duality [20-24] to non-gravitational field theories in one dimension fewer, at large $N$ (where $N$ is the rank of a field theory gauge group, or an analogue thereof). This is why our heat engines in this context are called "holographic". As pointed out in [1], since changing $\Lambda$ involves changing the $N$ of the dual theory, the heat engine cycle is a kind of tour on the space of field theories rather than staying within one particular field theory (as pointed out in [25] it also involves changing the size of the space the field theories live on). It is currently unclear as to the precise application of these heat engines in this 
context or any other, but as defined above they are certainly rich and well-defined enough in their own right to warrant an exploration of their physics.

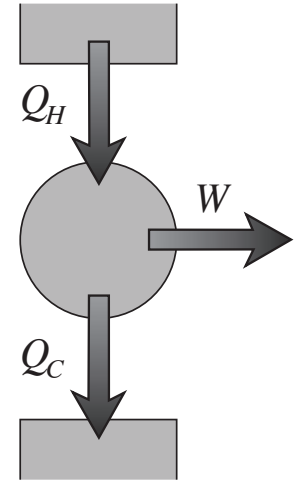

(a)

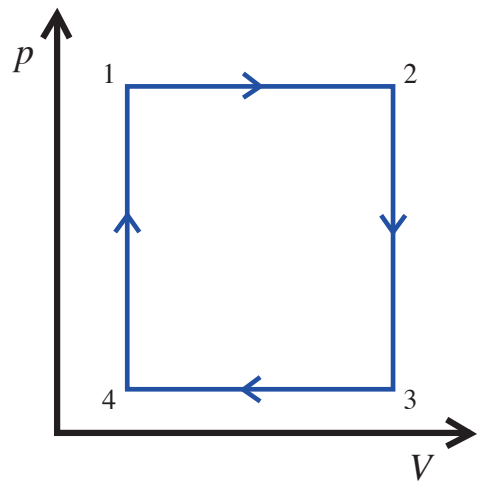

(b)

Figure 1. (a) The flows in a cycle; (b) A special cycle.

For now, focus on the cycle given in Figure 1b. In [1], it is explained why such a choice is natural for static black holes, which we study here $([18,19,26-29]$ have since done further studies of such heat engines). The work performed is $W=\left(V_{2}-V_{1}\right)\left(p_{1}-p_{4}\right)$, where the subscripts refer to the quantities evaluated at the corners labeled $(1,2,3,4)$. In the absence of scalars, Isochors are also adiabats for static black holes (for the STU black holes, for example, it was shown in [28] that the entropy and volume are independent functions, we thank an anonymous referee for suggesting this clarification), and so the heat flows take place entirely along the top and bottom, with the upper isobar giving the net inflow of heat and the lower isobar giving the exhaust:

$$
Q_{H}=\int_{T_{1}}^{T_{2}} C_{p}\left(p_{1}, T\right) d T, \quad Q_{C}=\int_{T_{4}}^{T_{3}} C_{p}\left(p_{4}, T\right) d T,
$$

where the specific heat at constant pressure $C_{p} \equiv T \partial S /\left.\partial T\right|_{p}$.

In the previous work on various black hole examples, the resulting efficiency $\eta=W / Q_{H}$ was evaluated in a high temperature limit, since in general, the relations between $S$ and $T$ are such that the $T$-integral needed to evaluate $Q_{H}$ is difficult to perform exactly. $[18,19]$ organize the computations of all needed quantities by working in terms of the horizon radius $r_{+}$, treating it as an independent parameter in terms of which all quantities can be most easily written. The high temperature expansions of all quantities were then developed by working out the high temperature expansion of $r_{+}(T)$. It is worth noting here that a next logical step could be to recast the $T$-integrals in Equation (1) as $r_{+}$-integrals. Since $C_{p}\left(r_{+}\right)$is (for some classes of black hole) a ratio of polynomials in $r_{+}$, depending upon the nature of the $\partial r_{+} / \partial T$ Jacobian this might result in simpler expressions for the efficiency (this was also noted by Shao-Wen Wei, in a private communication). Indeed, as can be seen by evaluating a few examples, this is correct. In fact, there's a much simpler way of looking at the whole picture that results in simple expression for the efficiency that makes it easy to see exactly why this works. We will explore this next. 


\section{A Simple Efficiency Formula}

The key point is that when black holes are the focus, it is the enthalpy $H$ that takes center stage in the First Law of Thermodynamics, since it is identified with the mass $M$ of the black hole [2]. So instead of writing the First Law in terms of the internal energy, $d U=T d S-p d V$, one writes:

$$
d H=T d S+V d p
$$

and since our heat flows are along isobars, for which $d p=0$, we see immediately that our total heat flow $\int T d S$, regardless of which variable we care to write it in terms of, is simply the enthalpy change, which is just the change in the black hole mass $M$. Therefore, we have a remarkably simple way to write our efficiency formula entirely in terms of the black hole mass evaluated at the corners:

$$
\eta=1-\frac{M_{3}-M_{4}}{M_{2}-M_{1}}
$$

The mass $M$ is most naturally written as a function of $r_{+}$and $p$. Depending upon which parameters of the cycle are prescribed (e.g., Scheme $1\left(T_{1}, T_{2}, p_{1}, p_{4}\right)$ or Scheme $2\left(T_{1}, T_{4}, V_{1}, V_{3}\right)$ in $[18,19])$, the equation of state can be readily used to evaluate the $r_{+, i}$ and hence the mass values. In fact, if the parameters specified are all pressures and volumes (e.g., $\left.\left(p_{1}, p_{4}, V_{1}, V_{2}\right)\right)$, the equation of state is not needed at all, (since the volume $V$ is a simple function of $r_{+}$) and the resulting exact efficiency formula is remarkably simple.

\subsection{Examples}

A nice class of examples is the static black hole of charge $q$ in $D$ dimensions that has mass formula:

$$
M=\frac{(D-2) \omega_{D-2}}{16 \pi}\left(\alpha r_{+}^{D-5}+r_{+}^{D-3}+\frac{q^{2}}{r_{+}^{D-3}}+16 \pi p \frac{r_{+}^{D-1}}{(D-1)(D-2)}\right),
$$

where for $\alpha=0$ we are in Einstein-Maxwell gravity, and for non-zero $\alpha$ we are in the Gauss-Bonnet generalization (see $[18,30]$ for details and background; note that the Gauss-Bonnet extension is only non-trivial for $D>4$ ). The relationship between $T, r_{+}$and $p$ is given by:

$$
T=\frac{1}{4 \pi r_{+}\left(r_{+}^{2}+2 \alpha\right)}\left(\frac{16 \pi p r_{+}^{4}}{(D-2)}+(D-3) r_{+}^{2}+(D-5) \alpha-(D-3) \frac{q^{2}}{r_{+}^{2 D-8}}\right) .
$$

There is no need to work at high temperature, as our Formula (3) is exact. In fact, it is pleasing to see how the high temperature expansion is corrected by it. In [18], for a given chosen (large) temperature, the expansions begin to break down once $\alpha$ gets too large. Using the formula to compute the exact efficiency is straightforward, and if (for example) $T_{1}, T_{2}, p_{1}$ and $p_{4}$ are specified parameters, one can invert Equation (5) to compute the radii $r_{+, 1}=r_{+, 4}$ and $r_{+, 2}=r_{+, 3}$, and compare to the result for the large $T$ procedures done in [18]. Such an example is presented in Figure 2. As $\alpha$ increases, the deviation from accuracy (the upper curve is the large $T$ result) grows since the large $T$ expansion under-estimates the heat and over-estimates the work, as can be determined by a direct comparison of those quantities with the exact result. 


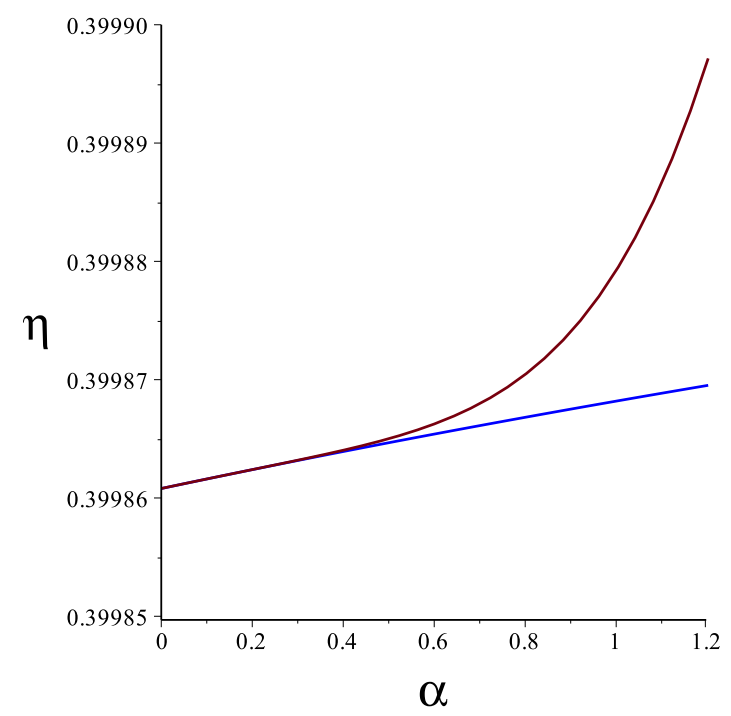

Figure 2. Comparison of the high temperature result for the efficiency (upper curve), at fixed working temperatures, to the exact result (lower curve), as the Gauss-Bonnet parameter $\alpha$ is increased. Here we chose parameters $q=0.1, T_{1}=50, T_{2}=60, p_{1}=5$ and $p_{4}=3$.

\section{The Efficiency for Arbitrary Cycles}

Working out the efficiency of more general shapes of cycle is in principle difficult, since the specific heat along the cycle is in general quite complicated, even for static black holes. Computing the heat flows would be quite a challenge. A remarkable and immediate consequence of our result Equation (3) is that it can be used as the basis for an algorithm for computing the efficiency of a cycle of arbitrary shape to any desired accuracy. The following describes how: Cycles are additive, in the sense indicated in Figure 3, and any closed shape in the $(p, V)$ plane can be approximated by tiling with a regular lattice of rectangles.

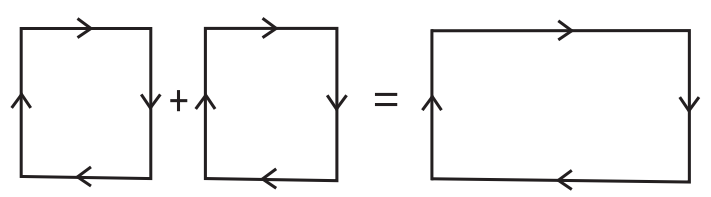

Figure 3. Adding cycles that share an edge.

There will of course be parts where the tiling's edge does not quite match the edge of the cycle's contour. The amount of the failure can be reduced by simply shrinking the size of the unit cell. At any given desired degree of accuracy (cell size), the efficiency is computed simply as follows: Label all cells at the corners as was done for the prototype cycle in Figure 1b. Only the cells at the edge contribute. They do so by either having their upper edge open (no adjoining cell), in which case we call it a hot cell, or by having their lower edge open, a cold cell. Summing all the hot cell mass differences (evaluated at the top edges) will give $Q_{H}$ and summing all the cold cell mass differences (evaluated at the bottom edges) will yield $Q_{C}$, and the efficiency follows:

$$
\eta=1-\frac{Q_{C}}{Q_{H}}, \quad Q_{H}=\sum_{i \text { th hot cell }}\left(M_{2}^{(i)}-M_{1}^{(i)}\right), \quad Q_{C}=\sum_{i \text { th cold cell }}\left(M_{3}^{(i)}-M_{4}^{(i)}\right)
$$

\subsection{A Triangular Example}

A triangular cycle was chosen to illustrate the algorithm in action. It has two edges parallel to the axes and one sloping edge in cycling from $(p, V)$ values $(2,30)$ to $(1,30)$ to $(1,10)$ and returning. 
One can choose to break it into a staircase-like rectangular lattice tiling that has $N$ rectangles (not to be confused with the $N$ of Section 1) approximating the sloped edge, with better accuracy at larger $N$, as in Figure 4.

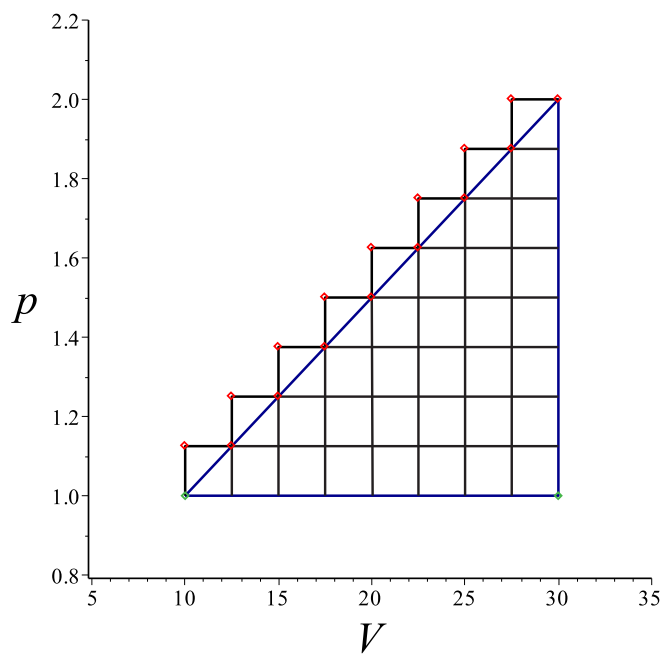

(a)

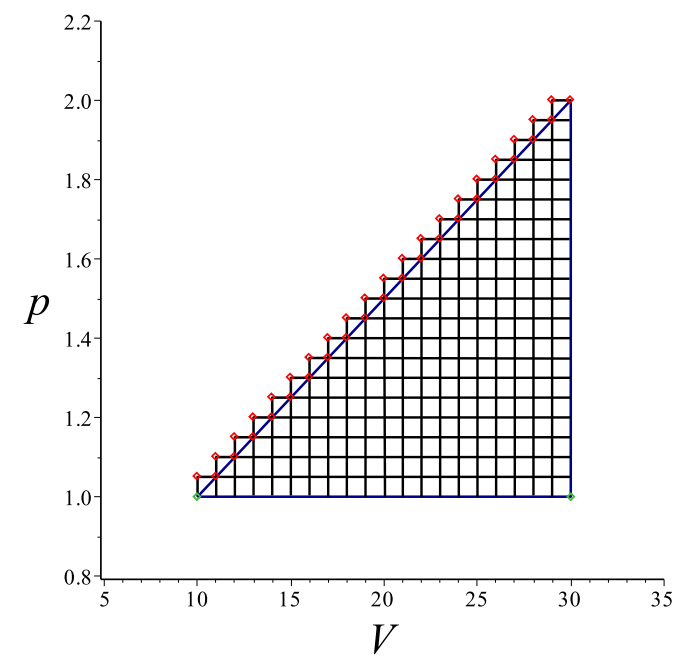

(b)

Figure 4. (a) The discretized triangle with $N=8$; (b) The discretized triangle with $N=20$. The red dots mark the (upper) horizontal segments along which $Q_{H}$ is computed, and the green dots mark the (lower) horizontal segment along which $Q_{C}$ is computed. See text for discussion.

A simple procedure was coded for the computation of the required $r_{+}$and $p$ values for each cell, summing the contributions to $Q_{H}$ according to our Formula (6). In this case $Q_{C}$ comes from the bottom edge as the overall mass difference along the base. Einstein-Maxwell black holes were used (i.e., $\alpha=0$ ), choosing the case $D=5$ in Equation (4) for the mass. Figure 5 shows the rapid convergence toward the result as $N$ is increased.

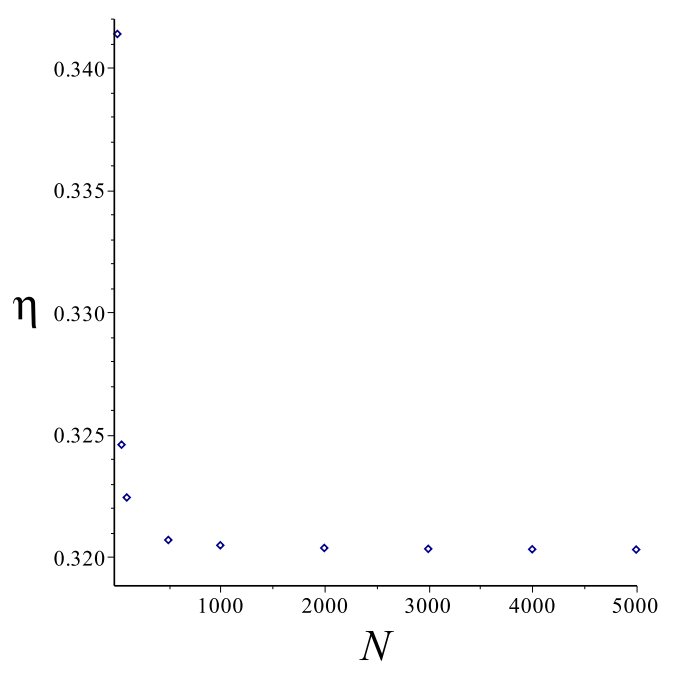

Figure 5. The convergence to the efficiency $\eta \sim 0.32027$ as the discretizations of the triangle become more accurate as $N$ increases. See text for discussion, and Figure 4 . Successive values include 0.34136, $0.32243,0.32070$, and 0.32030 for $N=10,100,500$, and 5000 respectively. 


\section{Closing Remarks}

A remarkably simple Expression (3) for the efficiency of a rectangular cycle for a class of static black holes was presented, simplifying earlier presented expressions and allowing it to be computed exactly. The whole formula is in terms of the black hole mass evaluated at each of the four corners of the cycle. Since the mass is often readily computable in a closed form expression, this is an extremely compact result. Section 2.1 compared the results to the high temperature expansions obtained in earlier work.

While it might seem relevant only to a special class of cycle, the expression turns out to be quite powerful, being the seed of an algorithm for computing the efficiency of cycles of arbitrary shape using a well-defined geometrical procedure given in Section 3. Such shapes would in general be hard to compute the efficiency for by, e.g., numerical integration methods along the path. The presented example (the triangle of Section 3.1) shows how rapidly the algorithm converges to a result for the efficiency.

In fact, the procedure extends to wider classes of heat engine, whether a black hole is used as a working substance or not. The key object to be able to compute with is the basic unit cell needed to tile a cycle of arbitrary shape. More generally, the unit cell is actually a Brayton/Joule cycle, composed of two isobars and two adiabats. (In this paper it is a rectangle, since for static black holes (without scalars), adiabats are isochors). So all the heat flows are taken into account on the top and bottom edges. Since they are isobars, the heat flows can be written entirely in terms of the enthalpy. So for any system for which one can readily compute the enthalpy $H$ (and black holes turn out to be such a system since $H$ is simply the mass $M$ ), and for which one knows the adiabatic curves, the same geometrical algorithm can be used to compute the efficiency of an arbitrary cycle in the manner described in Section 3. It would be interesting to study some examples of this application.

Acknowledgments: Clifford V. Johnson would like to thank the US Department of Energy for support under grant DE-FG03-84ER-40168, and Amelia for her support and patience.

Conflicts of Interest: The author declares no conflict of interest.

\section{References}

1. Johnson, C.V. Holographic Heat Engines. Class. Quant. Grav. 2014, 31, 205002, arXiv:hep-th/1404.5982.

2. Kastor, D.; Ray, S.; Traschen, J. Enthalpy and the Mechanics of AdS Black Holes. Class. Quant. Grav. 2009, 26, 195011, arXiv:hep-th/0904.2765.

3. Caldarelli, M.M.; Cognola, G.; Klemm, D. Thermodynamics of Kerr-Newman-AdS black holes and conformal field theories. Class. Quant. Grav. 2000, 17, 399-420, arXiv:hep-th/hep-th/9908022.

4. Wang, S.; Wu, S.Q.; Xie, F.; Dan, L. The First laws of thermodynamics of the (2+1)-dimensional BTZ black holes and Kerr-de Sitter spacetimes. Chin. Phys. Lett. 2006, 23, 1096-1098, arXiv:hep-th/hep-th/0601147.

5. Sekiwa, Y. Thermodynamics of de Sitter black holes: Thermal cosmological constant. Phys. Rev. D 2006, 73, 084009, arXiv:hep-th/hep-th/0602269.

6. Larranaga Rubio, E.A. Stringy Generalization of the First Law of Thermodynamics for Rotating BTZ Black Hole with a Cosmological Constant as State Parameter. 2007, arXiv:gr-qc/0711.0012.

7. Dolan, B.P. The cosmological constant and the black hole equation of state. Class. Quant. Grav. 2011, 28, 125020, arXiv:gr-qc/1008.5023.

8. Cvetic, M.; Gibbons, G.; Kubiznak, D.; Pope, C. Black Hole Enthalpy and an Entropy Inequality for the Thermodynamic Volume. Phys. Rev. D 2011, 84, 024037, arXiv:hep-th/1012.2888.

9. Dolan, B.P. Compressibility of rotating black holes. Phys. Rev. D 2011, 84, 127503, arXiv:gr-qc/1109.0198.

10. Dolan, B.P. Pressure and volume in the first law of black hole thermodynamics. Class. Quant. Grav. 2011, 28, 235017, arXiv:gr-qc/1106.6260.

11. Henneaux, M.; Teitelboim, C. The Cosmological Constant as a Canonical Variable. Phys. Lett. B 1984, 143, $415-420$.

12. Teitelboim, C. The Cosmological Constant as a Thermodynamic Black Hole Parameter. Phys. Lett. B 1985, $158,293-297$. 
13. Henneaux, M.; Teitelboim, C. The Cosmological Constant and General Covariance. Phys. Lett. B 1989, 222 , 195-199.

14. Bekenstein, J.D. Black holes and entropy. Phys. Rev. D 1973, 7, 2333-2346.

15. Bekenstein, J.D. Generalized second law of thermodynamics in black hole physics. Phys. Rev. D 1974, 9, 3292-3300.

16. Hawking, S. Particle Creation by Black Holes. Commun. Math. Phys. 1975, 43, 199-220.

17. Hawking, S. Black Holes and Thermodynamics. Phys. Rev. D 1976, 13, 191-197.

18. Johnson, C.V. Gauss-Bonnet Black Holes and Holographic Heat Engines Beyond Large N. 2015, arXiv:hep-th/1511.08782.

19. Johnson, C.V. Born-Infeld AdS Black Holes as Heat Engines. 2015, arXiv:hep-th/1512.01746.

20. Maldacena, J.M. The large N limit of superconformal field theories and supergravity. Adv. Theor. Math. Phys. 1998, 2, 231-252, arXiv:hep-th/9711200.

21. Witten, E. Anti De Sitter space and holography. Adv. Theor. Math. Phys. 1998, 2, 253-291, arXiv:hep-th/ 9802150.

22. Gubser, S.S.; Klebanov, I.R.; Polyakov, A.M. Gauge theory correlators from non-critical string theory. Phys. Lett. B 1998, 428, 105-114, arXiv:hep-th/9802109.

23. Witten, E. Anti-de Sitter space, thermal phase transition, and confinement in gauge theories. Adv. Theor. Math. Phys. 1998, 2, 505-532, arXiv:hep-th/9803131.

24. Aharony, O.; Gubser, S.S.; Maldacena, J.M.; Ooguri, H.; Oz, Y. Large N field theories, string theory and gravity. Phys. Rept. 2000, 323, 183-386, arXiv:hep-th/9905111.

25. Karch, A.; Robinson, B. Holographic Black Hole Chemistry. JHEP 2015, 12, 073, arXiv:hep-th/1510.02472.

26. Belhaj, A.; Chabab, M.; El Moumni, H.; Masmar, K.; Sedra, M.B.; Segui, A. On Heat Properties of AdS Black Holes in Higher Dimensions. JHEP 2015, 05, 149, arXiv:hep-th/1503.07308.

27. Sadeghi, J.; Jafarzade, K. Heat Engine of black holes. 2015, arXiv:hep-th/1504.07744.

28. Caceres, E.; Nguyen, P.H.; Pedraza, J.F. Holographic entanglement entropy and the extended phase structure of STU black holes. JHEP 2015, 09, 184, arXiv:hep-th/1507.06069.

29. Setare, M.R.; Adami, H. Polytropic black hole as a heat engine. Gen. Rel. Grav. 2015, 47, 133.

30. Cai, R.G.; Cao, L.M.; Li, L.; Yang, R.Q. P-V criticality in the extended phase space of Gauss-Bonnet black holes in AdS space. JHEP 2013, arXiv:gr-qc/1306.6233.

(C) 2016 by the author; licensee MDPI, Basel, Switzerland. This article is an open access article distributed under the terms and conditions of the Creative Commons Attribution (CC-BY) license (http://creativecommons.org/licenses/by/4.0/). 